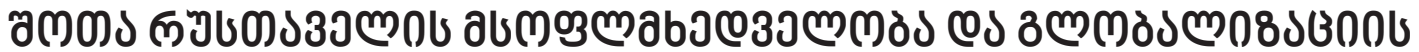

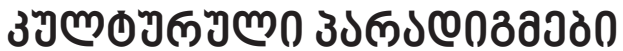

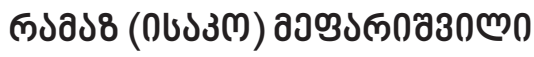

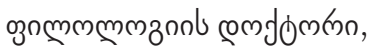

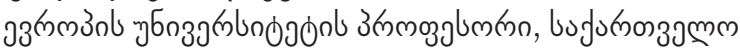

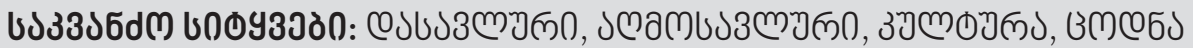

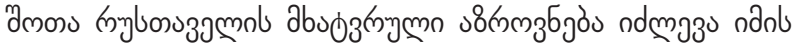

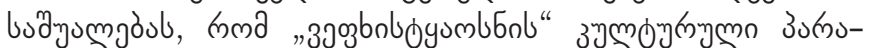

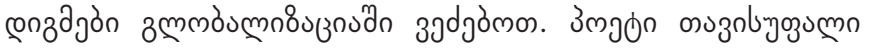

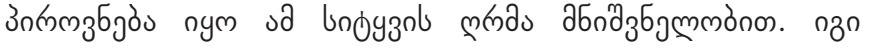

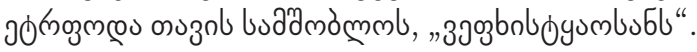

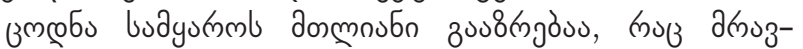

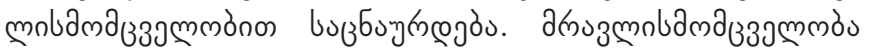

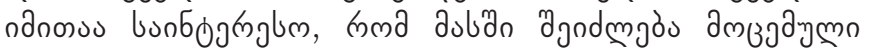

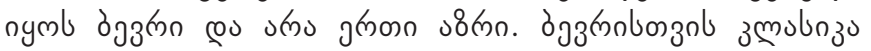

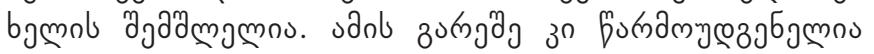

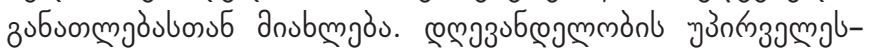

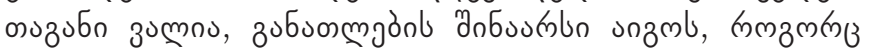

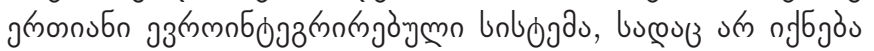

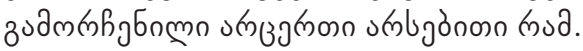

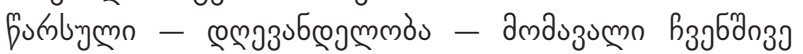

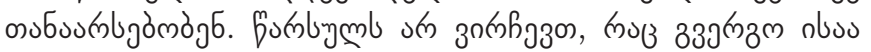

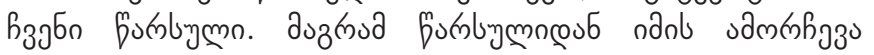

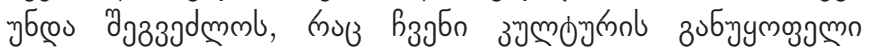

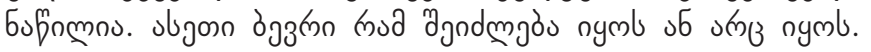

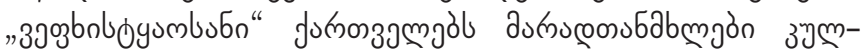

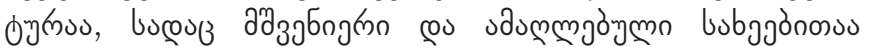

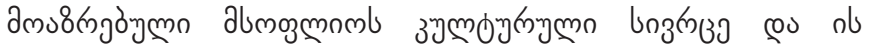

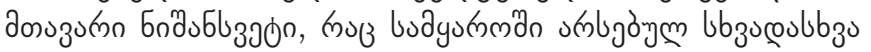

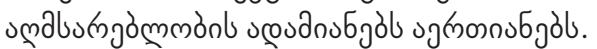

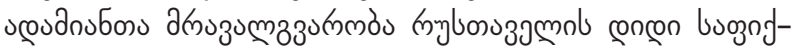

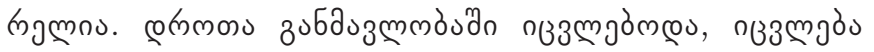

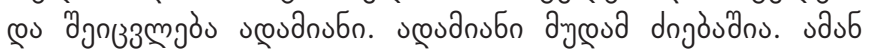

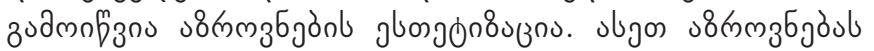

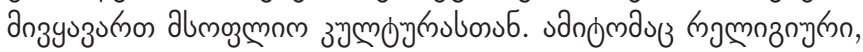

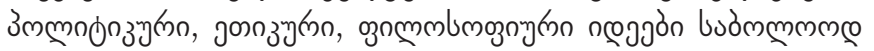

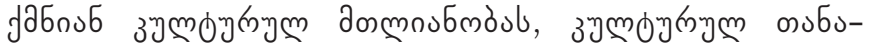

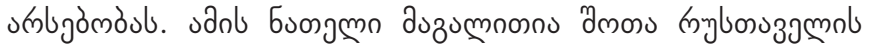

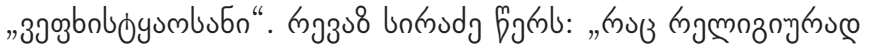

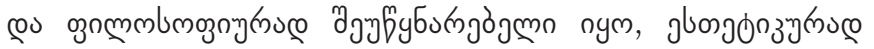

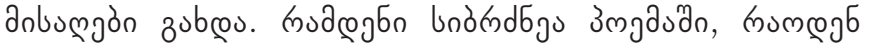
zuozumonm

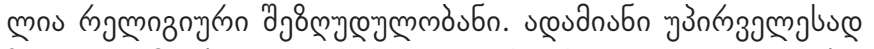

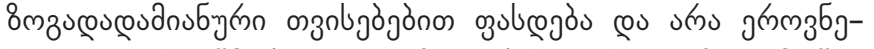

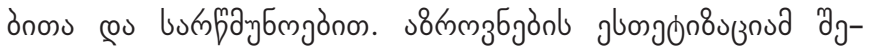

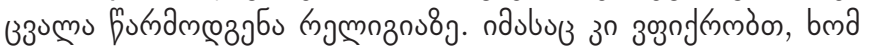

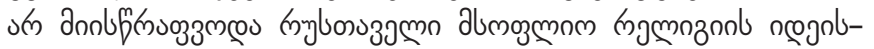

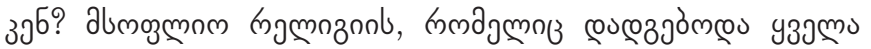

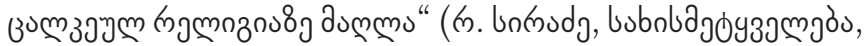

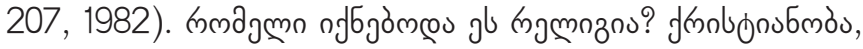

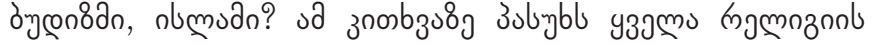

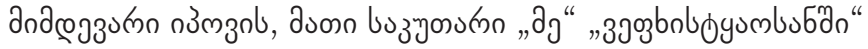
triglosszamol angén cosizmmos.

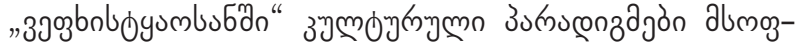
mamb donmnubmosubons

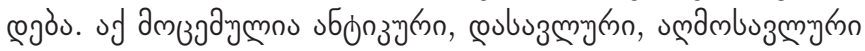

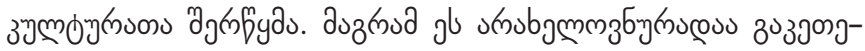

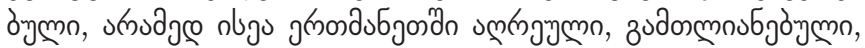

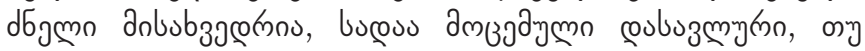

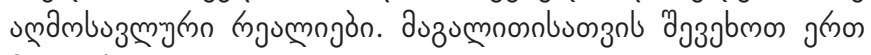
asonzubl.

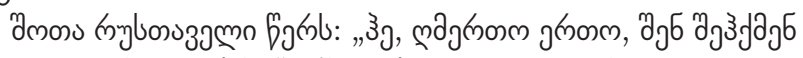

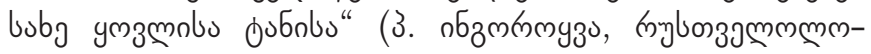

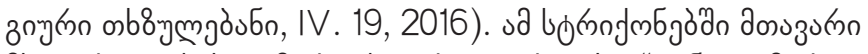

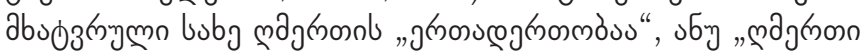

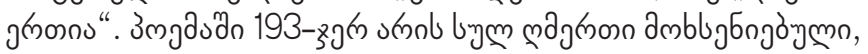

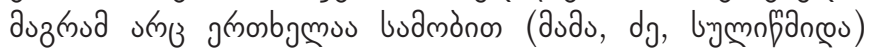

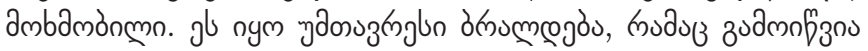

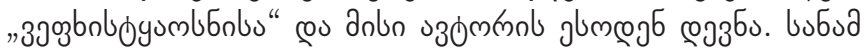

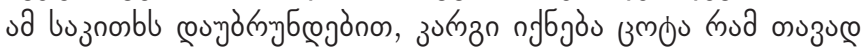

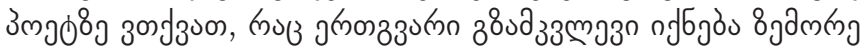
buznosbnl zubbnmzonbuonzol.

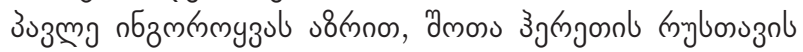

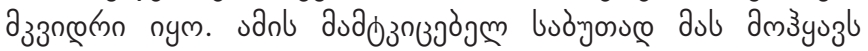

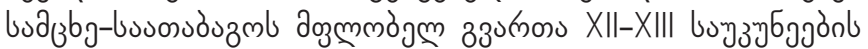
Eylubs, bunoug m m

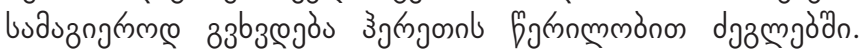

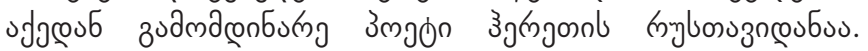

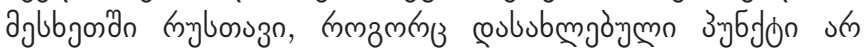

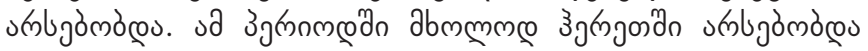
myloosozo.

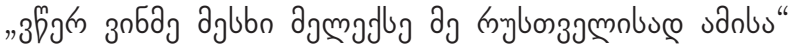

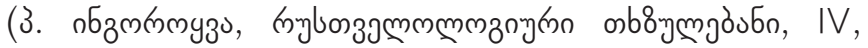

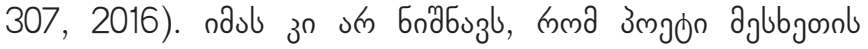

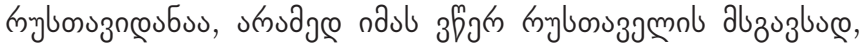

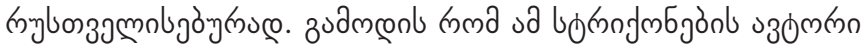

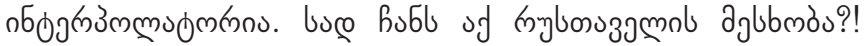

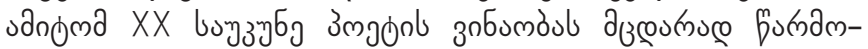
bobozos.

lym sbmubut coubringmes couznon zumazudznmols

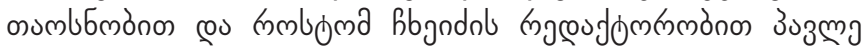

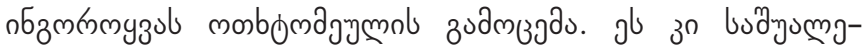

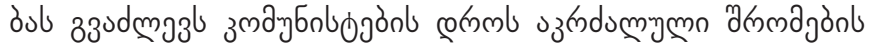

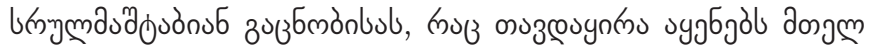




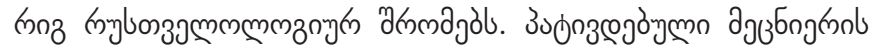

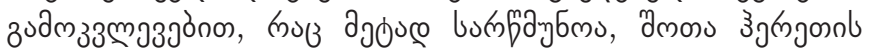

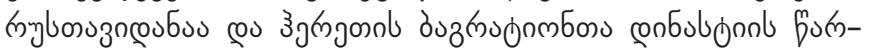

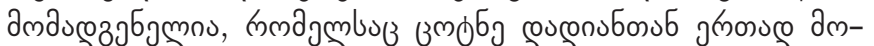

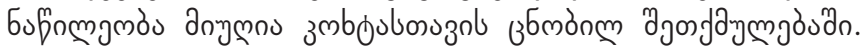

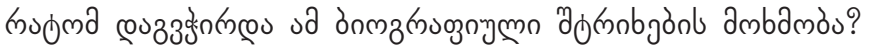

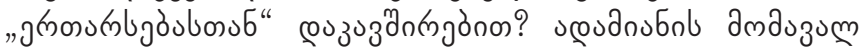

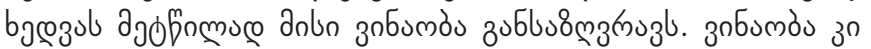

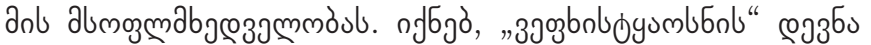

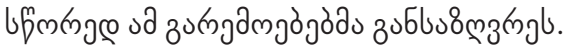

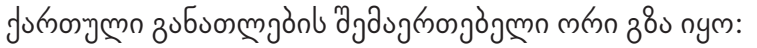

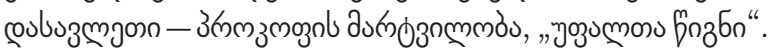

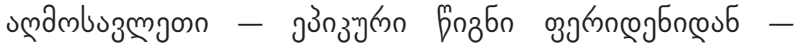

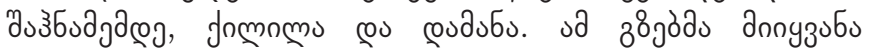

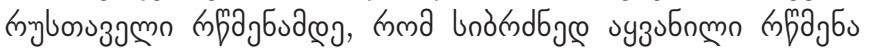

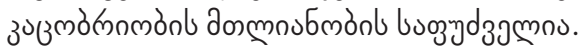

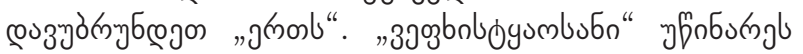

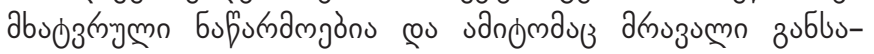

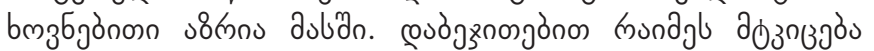

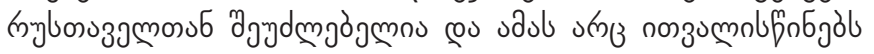
bjmmzб

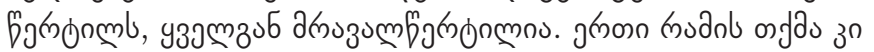

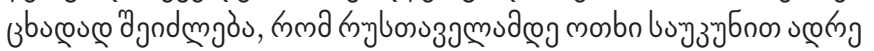

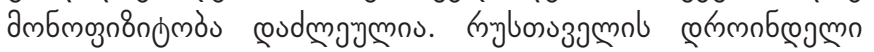

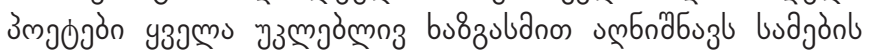

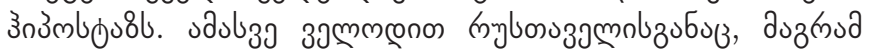

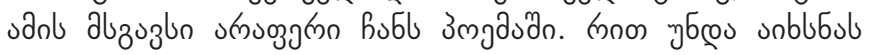

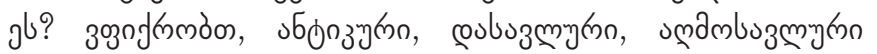

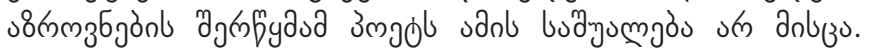

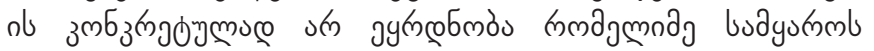

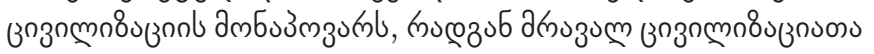

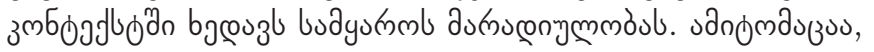

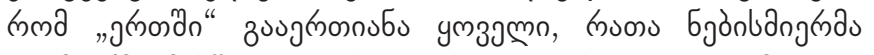
ง

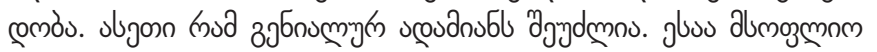

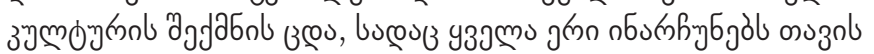

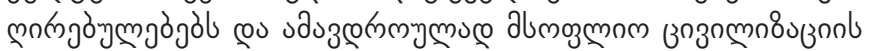

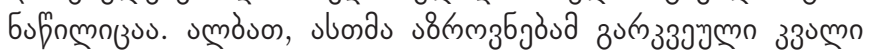

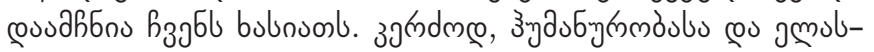
ony

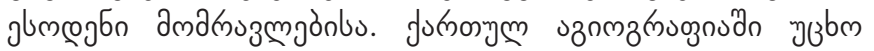

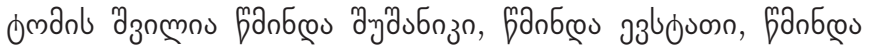

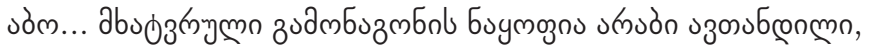

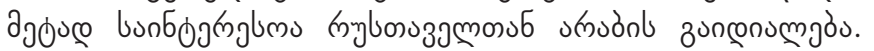

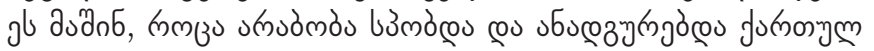

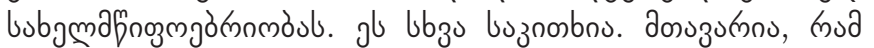

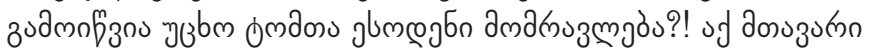

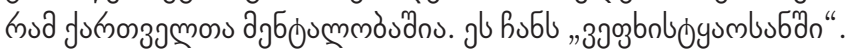

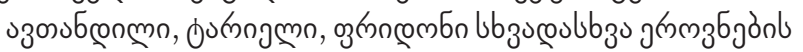

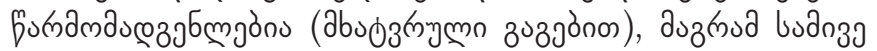

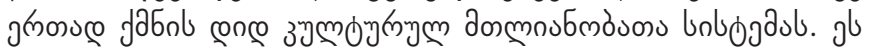

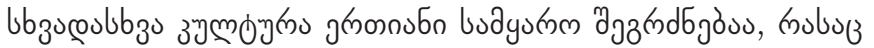

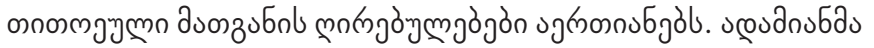

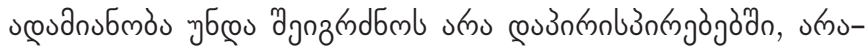

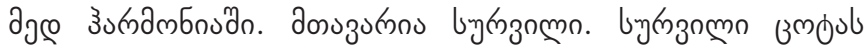

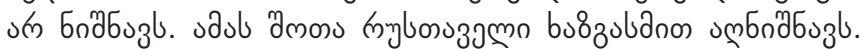

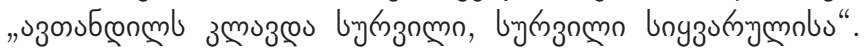

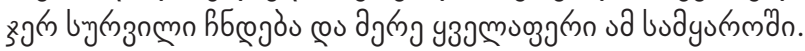

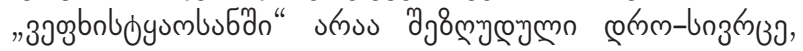

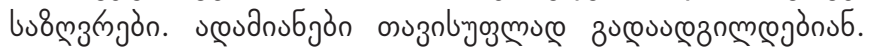

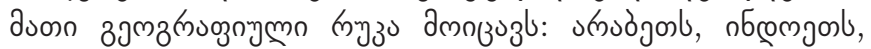

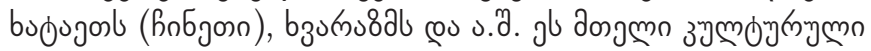

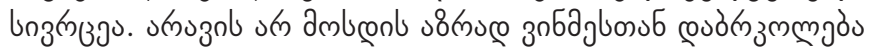

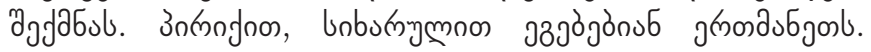

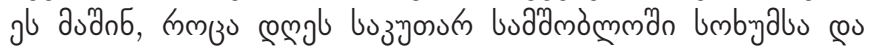

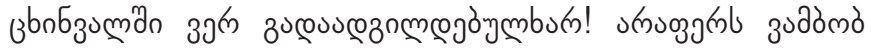

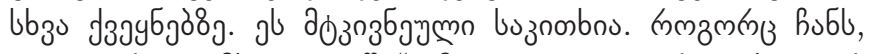

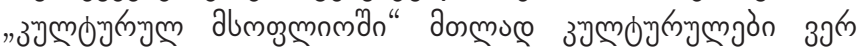

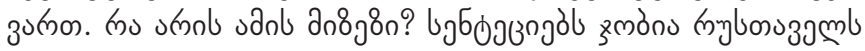

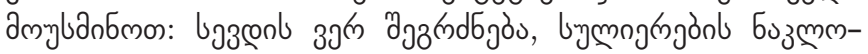

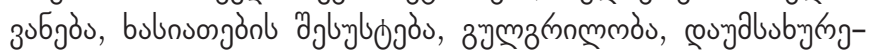

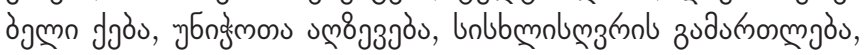

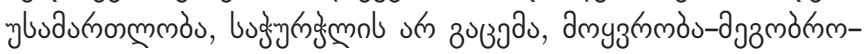
onl bmamo...

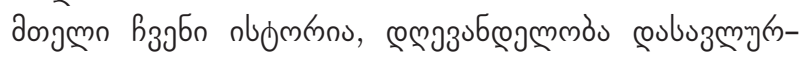

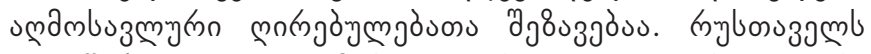

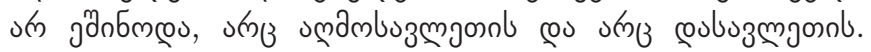

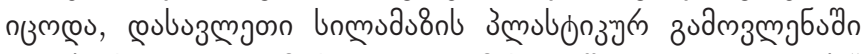

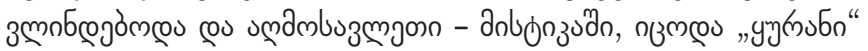

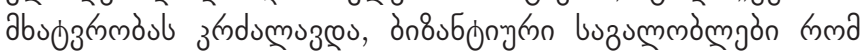

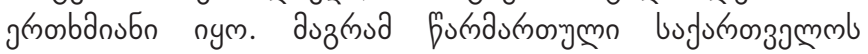

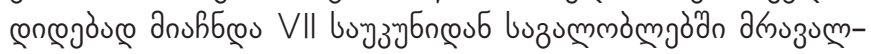

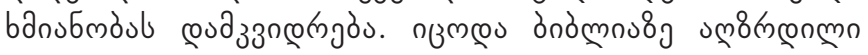

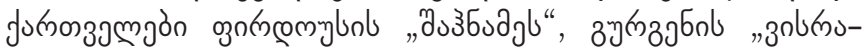

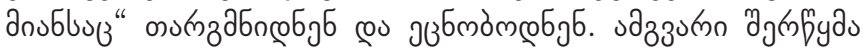

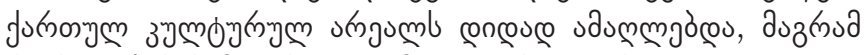

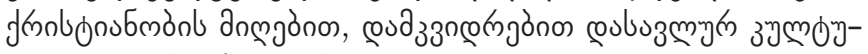

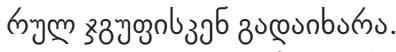

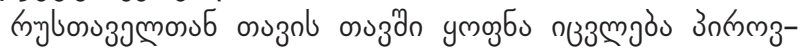

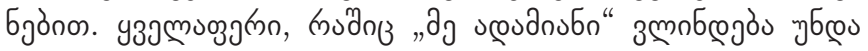

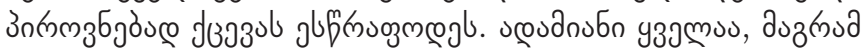

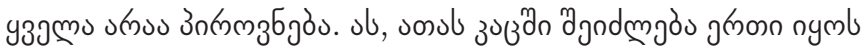

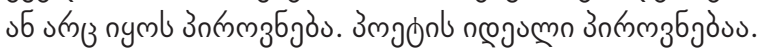

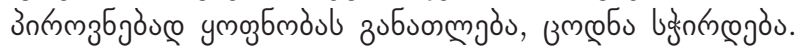

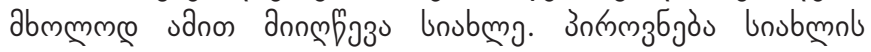

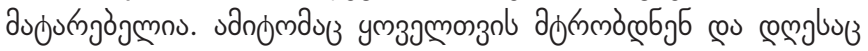

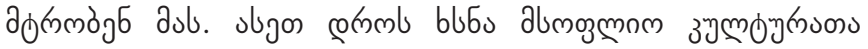

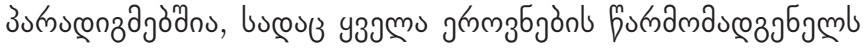

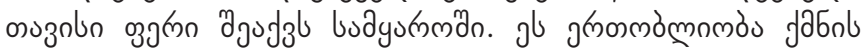

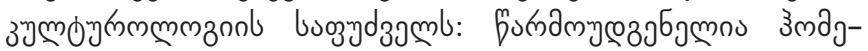
rmblol, bgr

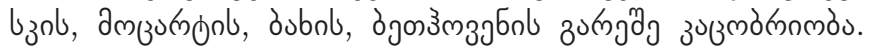

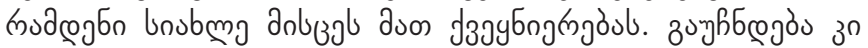

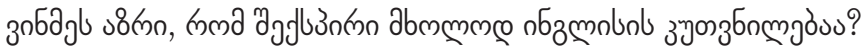

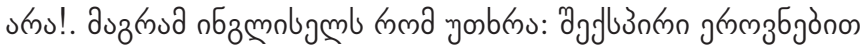

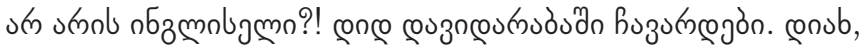




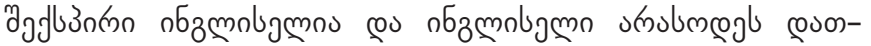

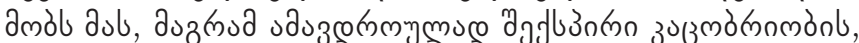

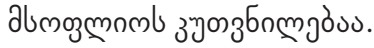

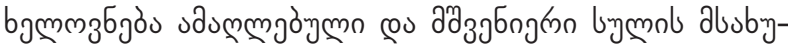

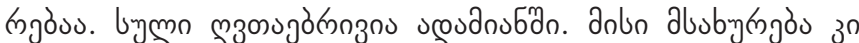

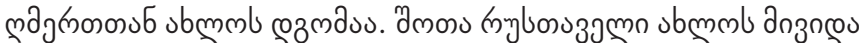

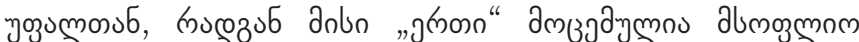

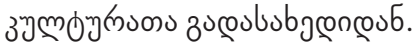

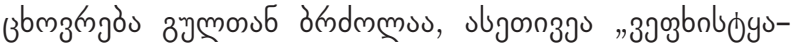

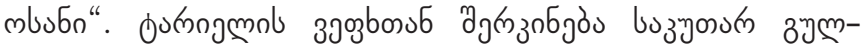

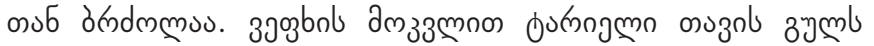

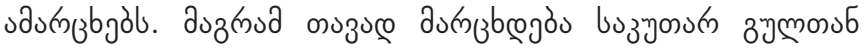

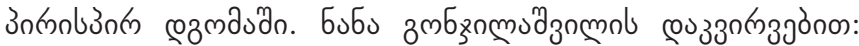

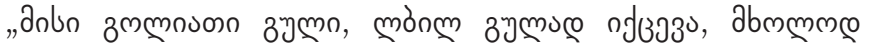

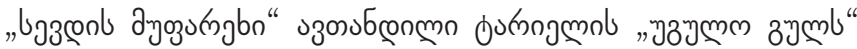

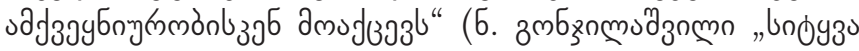

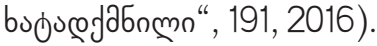

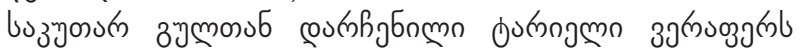

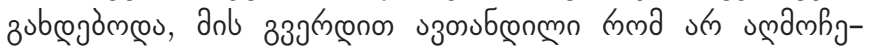

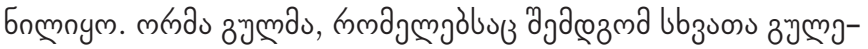

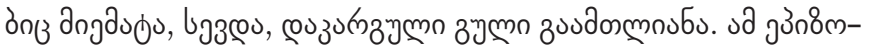

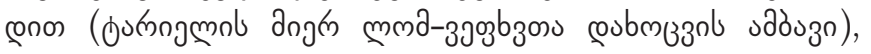

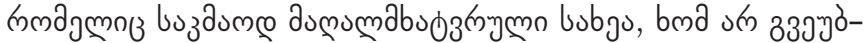

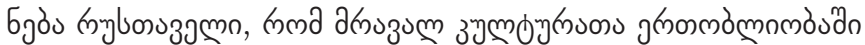

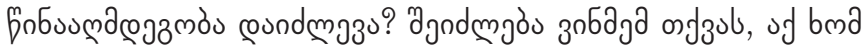

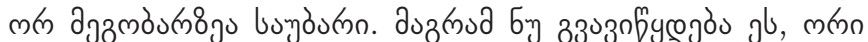

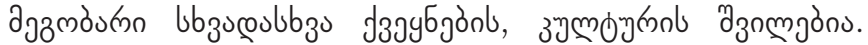

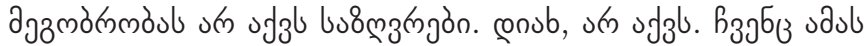

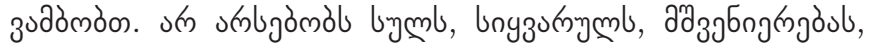

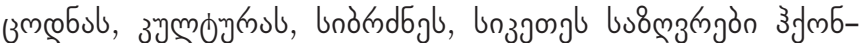
œ

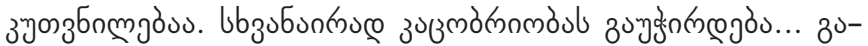

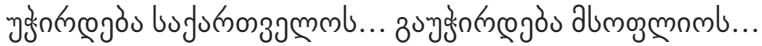

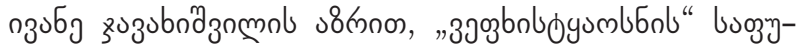

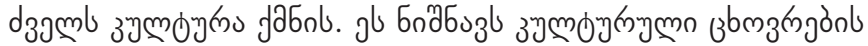

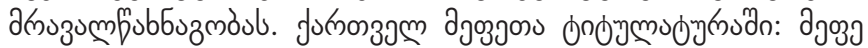

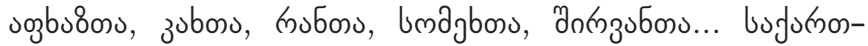

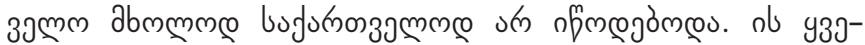

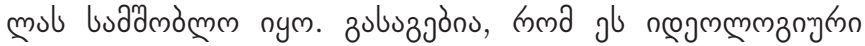

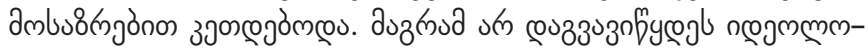

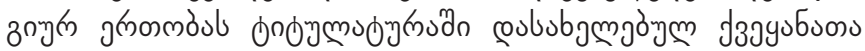

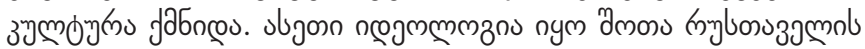

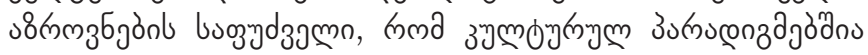

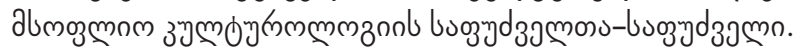

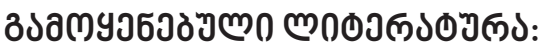

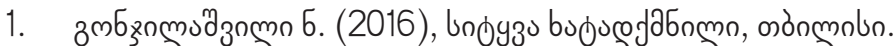

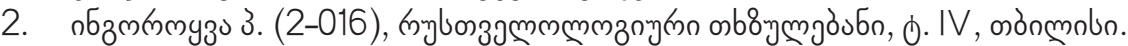

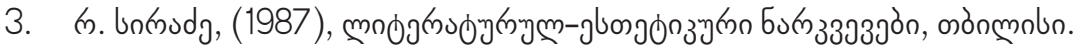

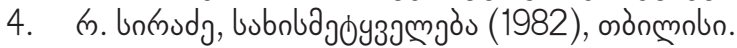




\section{SHOTA RUSTAVELI'S IDEOLOGY AND CULTURAL PARADIGMS OF GLOBALIZATION}

RAMAZ (ISAC) MEPARISHVILI

https://doi.org/10.35945/gb.2017.04.016

Doctor of Philology, Professor Of European University, Georgia

KEYWORDS: WESTERN, EASTERN, PERSONALITY, CULTURE, KNOWLEDGE

\section{SUMMARY}

According to the poem by Shota Rustaveli the human is the basic idea, a human has changed, changes and will change for different period of time. A human is always in search of something, this has caused aesthetics of thinking, this thinking leads us to world culture. That is why religious, political, ethical, philosophical ideas ultimately form the cultural integrity. Such a clear example is Shota Rustaveli's poem "The Knight in the Panther's Skin".

The core of the "Knight in the Panther's Skin" is a culture in which the diversity of cultural life is revealed. The concept of the poet's thinking comes from the cultural paradigm that underlies the world culture. 